\title{
Mycotic Cerebral Aneurysm and Septic Embolism Complicating Infective Endocarditis: A Case Report and Review of the Literature
}

\author{
M.A.Khouchab*, W.Belkho, H.Nabawi, M. El Jamili, N.Charei, S.El Karimi, M. El Hattaoui
}

Cardiology department, Mohamed VI University Hospital, Marrakech, Morocco

DOI: $10.36347 /$ sasjm.2020.v06i11.002

| Received: 02.11.2020 | Accepted: 12.11.2020 | Published: 16.11.2020

*Corresponding author: Mohamed Anas Khouchab

Abstract

Case Report

A 19-year-old male patient with history of recurrent untreated episodes of tonsillitis and acute rheumatic fever treated with benzathin benzylpenicillin presented to the hospital with high fever and diastolic murmur at the 3rd left intercostal space. Transthoracic and transesophageal echocardiography revealed vegetations on the aortic valve with severe aortic regurgitation. Three blood cultures were positive for Streptococcus Sinensis. Although antimicrobial therapy with a 3rd generation cephalosporin and aminoglycoside was effective for controlling infection, patient developed on the 13th day of hospitalisation headache and right leg intermittent claudication. Cerebral MRI showed right parietal lobe aneurysm with occipital microbleeds. A thrombosed aneurysm of $3,5 * 1,2 \mathrm{~cm}$ was shown to be present in the right tibialis posterior artery. Abdominal ultrasound and CT scan showed hepato-splenomegaly without any signs of embolism or infarction. This case confirms that infective endocarditis may present with various lifethreatening clinical situations. Clinicians should be aware that aortic valve vegetation induces generalized multi-organ embolism in the setting of IE in order to ensure prompt recognition and treatment of this fatal complication.

Keywords: Mycotic cerebral aneuvrysm, infective endocarditis, embolism.

Copyright $(92020$ The Author(s): This is an open-access article distributed under the terms of the Creative Commons Attribution 4.0 International License (CC BY-NC 4.0) which permits unrestricted use, distribution, and reproduction in any medium for non-commercial use provided the original author and source are credited.

\section{INTRODUCTION}

Infective endocarditis (IE) is associated with high hospital mortality, remaining a challenge for clinical diagnosis. It is characterized by the deposition of platelets, pathogens, and fibrin thrombi on valvular structures, which are prone to embolism and most commonly involved in cerebrovascular circulation. The most common symptom of IE is fever and cardiac symptoms[1]. Valvular vegetation is related to blood flow turbulence, inflammatory reactions, and injuries of the endothelial valve.

Despite advances in echocardiographic investigation, directed antibiotic therapy, and early surgical treatment, infective endocarditis remains an important cause of mortality among the infectious diseases. Therefore, its early identification is fundamental for reducing its complications and mortality.

\section{CASE REPORT}

A 19-year-old patient with history of recurrent untreated episodes of tonsillitis and acute rheumatic fever treated with benzathin benzylpenicillin 1,2 MUI/3 weeks, was admitted to the hospital wit high fever, bilateral and peripheral migratory arthralgias affecting large joints (knees and ankles), with asthenia. The patient denied previous intravenous drug abuse.

Physical examination revealed a temperature of $39^{\circ} \mathrm{C}$, a Grade IV diastolic murmur at the 3rd left intercostal space. Laboratory values were significant for a white blood cell count of 14000 cells/mm3, a CRP of $95 \mathrm{mg} / \mathrm{L}$, and an erythrocyte sedimentation rate of 104 $\mathrm{mm} / \mathrm{H}$. BUN, creatinine and serum electrolytes showed no abnormalities. Three blood cultures were positive for Streptococcus Sinensis.

Antibiotics were begun, 3rd generation cephalosporin (Ceftriaxone $2 \mathrm{~g} /$ day) and aminoglycoside (Gentamicin $160 \mathrm{mg} /$ day).

Transthoracic and transesophageal echocardiography revealed vegetations on the aortic valve, measuring $1,2 * 0,3 \mathrm{~cm}$ on the right coronary cusp, and $0,6 * 0,2$ on the non coronary cusp, with severe aortic regurgitation. Slit-lamp examination brought out the presence of Roth's spots in the eye fundus. 


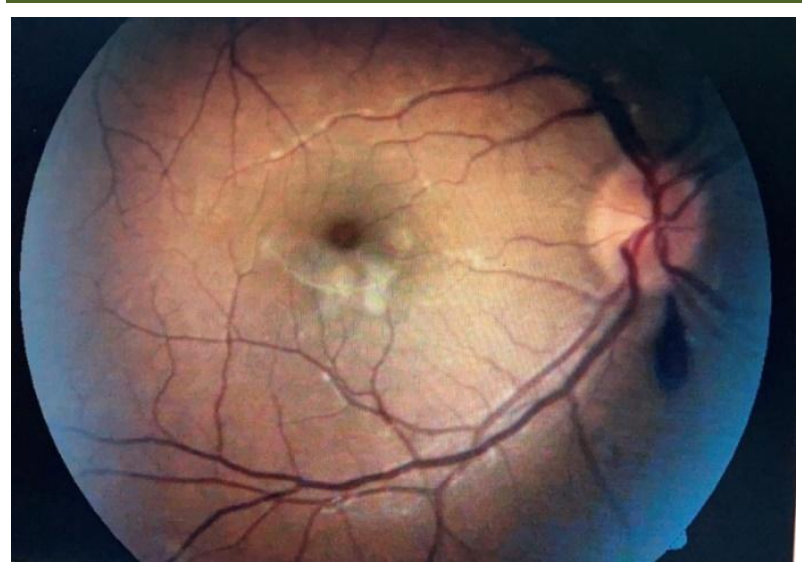

Fig-1: Slip lamp examination showing Roth's spots in the eye fundus

On the 13th day of hospitalisation, patient developed headache and right leg intermittent claudication. Cerebral MRI was realised and showed right parietal lobe aneurysm with occipital microbleeds. Lower extremity doppler ultrasound examination demonstrated a thrombosed aneurysm of $3,5^{*} 1,2 \mathrm{~cm}$ to be present in the right tibialis posterior artery. Abdominal ultrasound and CT scan revealed hepatosplenomegaly without any signs of embolism or infarction.

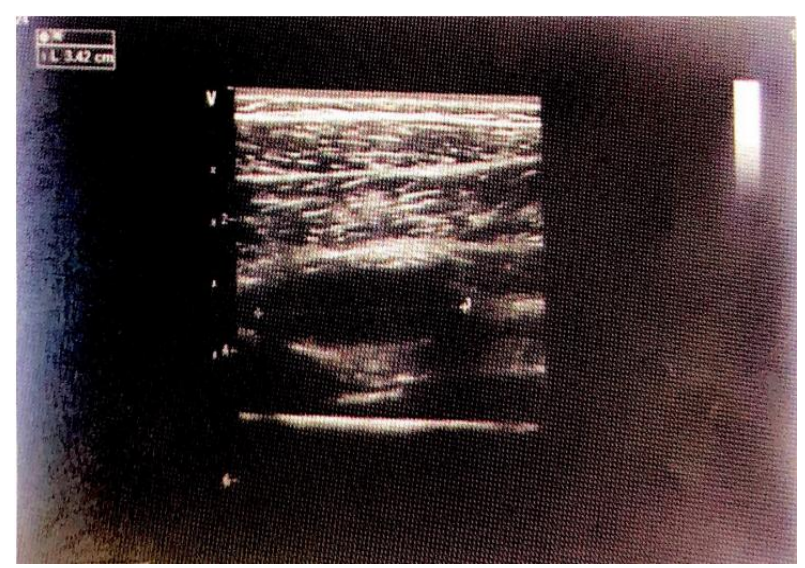

Fig-2: Doppler Ultrasound showing a thrombosed tibialis posterior aneurysm

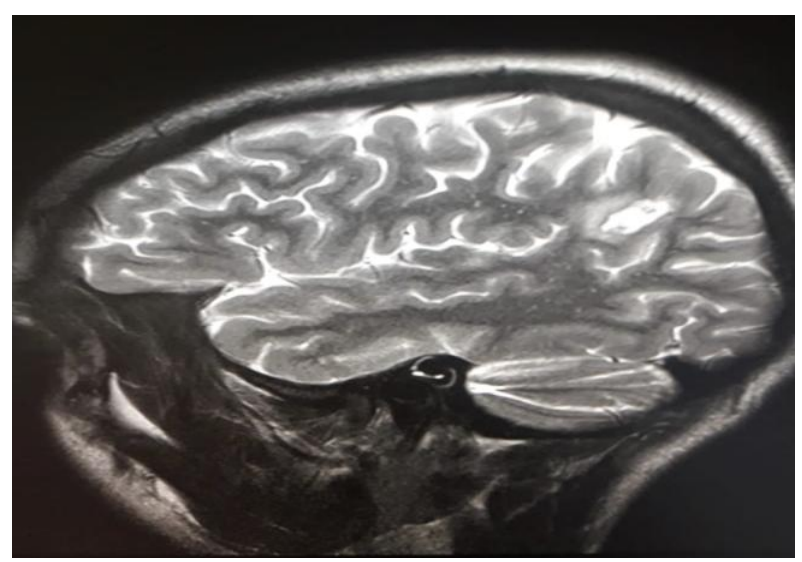

Fig-3: Cerebral MRI revealing mycotic aneurysm

\section{DISCUSSION}

In 1885 , Osler described that $80 \%$ of patients with bacterial endocarditis developed mycotic embolization with the potential for aneurysm formation in arteries particularly weakened by the infectious process [2]. Morbidity and mortality related to mycotic aneurysms, which at the time was not insignificant, has dramatically decreased with advances in antimicrobial therapy [3,4], and now they are almost exclusively limited to patients who use intravenous drugs.

The incidence of associated mycotic cerebral aneurysms in patients with infective endocarditis has been reported to be $2 \%$ [5].

Frazee et al. reported that the incidence of mycotic cerebral aneurysms represented $4 \%$ of all patients with intracranial aneurysms and $3 \%$ of all patients with infective endocarditis [6].

Among the causes of infective endocarditis, rheumatic and congenital heart disease have been considered. Also it has recently been reported that illicit intravenous drug abuse leads to increasing incidences of infective endocarditis in the West $[7,8]$.

The mortality rate of patients with mycotic cerebral aneurysm without rupture is $30 \%$, but $80 \%$ in cases where rupture does take place [9].

Also, a new aneurysm may appear subsequently. Due to these reasons, prompt and serial angiography should be done on patients with infective endocarditis who develop any neurological signs and symptoms, particularly headache or focal signs.

It is well-known that the treatment of infective endocarditis is adequate antibiotics therapy against the target organism and cardiac surgery such as valve replacement. However, some serious problems arise when infective endocarditis is combined with mycotic cerebral aneurysm.

In cases where infective endocarditis and mycotic cerebral aneurysm occur together, it must be decided which operation should be undertaken first, heart or brain. If cardiac surgery is performed prior to neurosurgery, systemic heparinization may lead to intracranial bleeding during cardiopulmonary bypass, and postoperative anticoagulant therapy may also lead to intracranial bleeding. If craniotomy is performed prior to cardiac surgery, the risk is high if the patient has heart failure. therefore, craniotomy should be considered before the patient develops severe heart failure.

If emergency valve replacement is necessitated, craniotomy should be considered shortly after cardiac surgery. A recent report described an endovascular technique for embolizing a mycotic 
cerebral aneurysm in a patient with infective endocarditis [10]. This method may be effective for some patients with severe heart failure.

On the other hand, lower extremity emboli may be the initial manifestation of infective endocarditis and can occur weeks to months after therapy is instituted. Lower extremity septic embolization may present either as acute limb threatening ischemia or as more indolent ischemia manifesting as claudication. Other less specific musculoskeletal symptoms may also occur.

In a retrospective review of 192 cases of bacterial endocarditis reported by Churchill and colleagues [11], 44\% of patients had complaints of arthralgias, arthritis, low back pain, or lower extremity myalgias. Just over half of these patients' symptoms were attributed to emboli to lower extremity muscle or skin.

Currently, the incidence of these aneurysms following an episode of endocarditis is quite rare and their location is dependent upon the peripheral lodgment site of mycotic emboli [12,13] These peripheral embolic events usually involve the lower extremities with most emboli lodging at the common femoral artery bifurcation.

Involvement of the arterial system below the femoral artery level is noteworthy and has been described only in case reports. To date, mycotic aneurysms distal to the femoral artery have been recorded at the popliteal artery (five patients), PTA (two patients), anterior tibial artery (one patient), tibioperoneal trunk (one patient), and the dorsalis pedis artery (one patient) [14].

In addition to a changé in associated morbidity, microbial etiology of mycotic aneurysms has shifted over time primarily on account of intravenous drug use. Prior to widespread antibiotic use, the involved organisms were typically gram positive cocci such as nonhemolytic streptococci, pneumococci, and staphylococci [15].

Similarly, staphylococci (aureus and epidermidis) and streptococci (viridans and faecalis) are cultured today from mycotic aneurysms in the nonintravenous drug abuse population [16].

However, in the intravenous drug-using population, polymicrobial infections are common. Gram negative organisms such as Pseudomonas aeruginosa are frequently cultured in addition to grampositive cocci. Fungi are also involved in a small but significant percentage of mycotic aneurysms and despite their infectious nature, up to $25 \%$ of mycotic aneurysms can be culture negative [16].
In contrast to the obvious need for antibiotic treatment in these cases, surgical management is less clear because of the paucity of cases in the literature.

Since there are no specific treatment guidelines, management is commonly guided by general principles of vascular surgery. Exigent hemorrhage necessitates acute ligation of the bleeding vessel with or without revascularization. Patent aneurysms should be excluded to prevent unwanted complications such as rupture, embolization, or acute thrombosis with resultant peripheral ischemia.

The choice of revascularization is dependent on aneurysm location and collateral flow. They are usually amenable to interposition vein graft replacement.

Prosthetic materials should be avoided because of the presence of active infection. In addition to the surgical intervention for the aneurysm, organismspecific intravenous antibiotic therapy should be instituted for a minimum of 6 weeks $[12,16]$. Treatment of asymptomatic thrombosed mycotic aneurysms should be individualized.

\section{CONCLUSION}

Despite modern clinical approaches, early infection-directed therapy, and better critical care capabilities, septic embolism continues to significantly affect the critically ill population. In addition to early clinical recognition, prompt identification of the underlying infectious source and immediate goaldirected antibiotic therapy are all critical to successful management of septic embolism. More widespread awareness of risk factors, clinical presentation, and management of septic embolism is needed in the modern intensive care unit.

\section{REFERENCES}

1. Unlu O, Lieber SB, Roman MJ. Mitral valve vegetation in antiphospholipid syndrome. Arthritis Rheumatol. 2016;68:1300.

2. Osler $\mathrm{W}$. The Gulstonian Lectures on malignant endocarditis.

3. Brown SL, Busuttil RW, Baker JD. Bacteriologic and surgical determinants of survival in patients with mycotic aneurysms. J Vasc Surg. 1984;1:541547.

4. Reddy DJ, Ernst CB. Infected aneurysms. In: Rutherford RB, ed. Vascular Surgery, 4th ed. Philadelphia: W.B. Saunders. 1995: 1139-1153.

5. Lerner PI. Neurologic complications of infective endocarditis. Med Clin North Am. 1985;65:385398.

6. Frazee JG, Cahan LD, Winter J. Bacterial intracranial aneurysms. J Neurosurg. 1980;53:633641. 
7. Cox SM, Hankins GDV, Leveno KJ, Cunningham FG. Bacterial endocarditis: a serious pregnancy complication. J Reprod Med. 1988;33:671-674.

8. Pastorek JG, Plauche WC, Faro S. Acute bacterial endocarditis in pregnancy: a report of three cases. J Reprod Med. 1983;28:611-614.

9. Bohmfalk GL, Story JL, Wissinger JP, Brown WE. Bacterial intracranial aneurysm. J Neurosurg. 1978;48:369-382.

10. Frizzell RT, Vitek JJ, Hill DL, Fisher III WS. Treatment of a bacterial (mycotic) intracranial aneurysm using an endovascular approach. Neurosurgery. 1993;32:852-854.

11. Churchill Jr MA, Geraci JE, Hunder GG. Musculoskeletal manifestations of bacterial endocarditis. Ann Int Med. 1977;87754-759.
12. Dean RH, Meacham PW, Weaver FA, Waterhouse G, O’Neil JA. Mycotic embolism and embolomycotic aneurysm: neglected lessons of the past. Ann Surg. 1986;204:300-307.

13. Guzzetta PC. Congenital and acquired aneurysmal disease. Semin Pediatr Surg. 1994;3:97-102.

14. Bonds JW, Fabian TC. Surgical treatment of mycotic popliteal artery aneurysm: a case report and review of the literature. Surgery. 1985;98:979982.

15. Osler W. The Gulstonian Lectures on malignant endocarditis. Br Med J. 1885;1:467.

16. Brown SL, Busuttil RW, Baker JD. Bacteriologic and surgical determinants of survival in patients with mycotic aneurysms. J Vasc Surg. 1984;1:541547. 